cause of hypertension, and early diagnosis does not carry the benefits that are claimed, for instance, in some forms of carcinoma. By far the greater need is the detection of undiagnosed hypertension, and a patient with primary aldosteronism treated with routine antihypertensive medication is not a medical disaster so long as the clinician is alive to the possibility of hypokalaemia in hypertensive patients.

${ }^{1}$ Conn JW, Cohen EL, Rovner DR, Nesbit RM. Normokalemic primary aldosteronism: a detectable cause of curable "essential" hypertension. f $A M A$ 1965;193:200-6.

2 Berecek KH, Bohr DF. Structural and functional changes in vascular resistance and reactivity in the deoxycorticosterone acetate (DOCA)hypertensive pig. Circ Res 1977;40, suppl 1:146-52.

3 Weinberger MH, Grim CE, Hollifield JW, et al. Primary aldosteronism. Ann Intern Med 1979;90:386-95.

4 Davies DL, Beevers DG, Brown JJ, et al. Aldosterone and its stimuli in normal and hypertensive man: are essential hypertension and primary hyperaldosteronism without tumour the same condition ? $\mathcal{F}$ Endocrinol $1979 ; 81: 279 \mathrm{p}-91 \mathrm{p}$.

${ }^{5}$ Biglieri EG, Schambelan M, Brust N, Chang B, Hogan M. Plasma aldosterone concentration: further characterization of aldosteroneproducing adenomas. Circ Res 1974 ;34, suppl $1: 183-91$.

${ }^{6}$ Ferriss JB, Brown JJ, Fraser R, et al. Results of adrenal surgery in patients with hypertension, aldosterone excess, and low plasma renin concentration. $\mathrm{Br}$ Med $\mathcal{F} 1975 ; \mathrm{i}: 135-8$.

7 Streeten DHP, Tomycz N, Anderson GH. Reliability of screening methods for the diagnosis of primary aldosteronism. Amer $\mathcal{f} \mathrm{Med}$ $1979 ; 67: 403-13$.

8 Ferriss JB, Neville AM, Brown JJ, et al. Hypertension with aldosterone excess and plasma-renin: pre-operative distinction between patients with and without adrenocortical tumour. Lancet 1970;ii:995-1000.

9 Luetscher JA, Gnaguly A, Melada GA, Dowdy AJ. Preoperative differentiation of adrenal adenoma from idiopathic adrenal hyperplasia in primary aldosteronism. Circ Res $1974 ; 34$, suppl $1: 175-82$.

10 Sarkar SD, Cohen EL, Beierwaltes WH, Ice RD, Cooper R, Gold EN. $A$ new and superior adrenal imaging agent 131-I-6 $\beta$-iodomethyl 19-nor-cholesterol. (NP-59): evaluation in humans. $\mathcal{F}$ Clin Endocrinol Metab 1977;45:353-62.

11 Clarke D, Johnston IDA, Wilkinson R, Hacking PM, Haggith JW. Severe hypertension in primary aldosteronism and good response to surgery. Lancet 1979 ;i :482-5.

12 Herwig KR. Primary aldosteronism: experiences with thirty-eight patients. Surgery $1979 ; 86: 470-3$.

${ }^{13}$ Herf SM, Teates DC, Tegtmeyer CJ, Vaughan ED, Ayers CR, Carey RM. Identification and differentiation of surgically correctable hypertension due to primary aldosteronism. Amer $\mathcal{F}$ Med 1979;67:397-402.

\section{Treating fungal infections}

A recently published symposium on the treatment of fungal infections in $\operatorname{man}^{1}$ opens with the statement that we still cannot control these satisfactorily. Nor did the contributions give hope of an early breakthrough; no really effective non-toxic antifungal has yet been established in clinical practice. A second factor that militates against predictable success in antifungal treatment is failure or delay in making the diagnosis, so that the available antimycotic agents are not used early and to their fullest effect.

Real skill in diagnostic mycology is confined to a few specialist centres, yet fungal infections present throughout hospital and community medicine. Part of the difficulty comes from their rarity: only dermatophyte infections and vaginal candidiasis are common, and clinicians have few opportunities to gain experience in the management of the other infections. Even with the two common conditions, however, failure of treatment and relapse is more frequent than is often acknowledged. ${ }^{2}$ An important principle restated in the symposium $^{3}$ is that isolation of Candida albicans from a perfectly healthy vagina is rare. Its presence in even small numbers (which may be a result of sampling error) is always an indication for treatment in the presence of symptomsand in pregnancy even in their absence.

Even in expert hands the methods for diagnosing disseminated fungal infection are often disappointingly unproductive. Despite the use of special media, which can be selected only if a tentative diagnosis has been reached, blood cultures fail to detect yeast-like fungi early enough to be useful. Lesions are often inaccessible: in the lung, for example, bronchial or open lung biopsy may be needed-procedures which may present formidable technical difficulties and carry unacceptable risks in patients having immunosuppressive treatment or with deficient platelets. With the notable exceptions of cryptococcosis and histoplasmosis reliable serological tests have not been developed. In candidiasis those available may be actively misleading. All in all, the delays and difficulties in the diagnosis of fungal infections are hardly surprising.

Even when the diagnosis is made (or in many cases when a decision is taken to try blind treatment) the available agents are less than ideal. Nevertheless, used early and with attention to detail they give better results than are achieved with haphazard use, prematurely curtailed for fear of toxicity. A fear of using amphotericin ${ }^{4}$ is still difficult to explain among physicians faced with potentially lethal infections in patients, most of whom have a serious predisposing disease. Amphotericin is the established antifungal drug for systemic use and has yet to be improved on for most purposes. It is undoubtedly toxic, producing fevers, rigors, venous thrombosis, and potassium-losing nephritis requiring the use of potassium supplements; but by using moderate dosage and hydrocortisone cover it may be given successfully. Treatment needs to be maintained for weeks and even months, a fact frequently not recognised. Such a regimen is more readily achieved with moderate daily doses (not exceeding $0.5 \mathrm{mg} / \mathrm{kg}$ ) than with high doses on alternate days or rapid infusion, both carrying a higher risk of side effects. ${ }^{5}$

The symposium refers to work $^{6}$ showing that the slow, stepwise increase in dose recommended by the makers fails to establish early therapeutic concentrations. Moderate dosage from the onset should be considered in severely ill patients, in whom the more cautious approach may lead to failure of treatment. Combination of amphotericin with flucytosine has become standard practice in the management of disseminated infection with yeast-like fungi. ${ }^{7-9}$ Treatment with flucytosine alone is not satisfactory since it leads to selection of resistant minority populations..$^{10-12}$ The combination may allow the use of reduced amounts of amphotericin, though the $20 \mathrm{mg}$ daily originally recommended may be too low. The amphotericin enhances the toxic effects of flucytosine on the bone marrow, giving thrombocytopenia and neutropenia, a complication which may be minimised ${ }^{5}$ by maintaining serum concentrations of flucytosine below $100 \mathrm{mg} / \mathrm{l}$. Even when used early and in combination treatment may fail, particularly in immunocompromised patients, who tend to suffer more acute infections. ${ }^{13}$

Disseminated fungal infection, especially cryptococcal meningitis, occurs almost exclusively in this group. In these patients intrathecal amphotericin is always necessary at the start of treatment and not, as one contributor ${ }^{14}$ implies, only in the face of relapse, itself a very bad prognostic feature. A difficult decision which will also have to be faced early is whether to attempt to reach organisms in the basal meninges and ventricles by cisternal puncture or the use of a reservoir.

The place of new antifungal drugs now available or being developed is difficult to delineate. The greatest deficiency 
in our present armament is in treating infections caused by filamentous fungi, Aspergillus and the phycomycetes, and there is little evidence that the newer compounds are better than amphotericin or even as good.

Most effort has been given to the development of the imidazoles-clotrimazole, miconazole, econazole, and ketoconazole. Clotrimazole has good activity against filamentous fungi as well as yeasts when given by mouth but it has proved too toxic to be marketed for systemic use. It is possibly the best topical antifungal agent now available (though in the dermatophyte infections systemic griseofulvin still has a paramount part to play). ${ }^{15}$ The other imidazoles, including miconazole, still require further systemic evaluation, as many of the contributors to the symposium emphasised. Miconazole is effective in coccidioidomycosis, a rare condition in Britain. When it is given by mouth blood concentrations of the drug are low and variable, and it is not active by this route in generalised candidiasis, though this is implied in one of the papers. In systemic infection miconazole has to be given parenterally, and no clear impression has yet emerged of its efficacy. In view of the evidence from laboratory studies of antagonism when combined with amphotericin ${ }^{16}$ the two should not be used together. Ketoconazole when given by mouth seems to be absorbed more reliably but unfortunately its activity against Aspergillus species is variable and overall low. ${ }^{17}$ Clearly much more work remains to be done on the imidazoles.

In the mean time, the best prospect for systemic mycoses remains the early use of amphotericin by all appropriate routes, and for this we urgently need better techniques for early diagnosis. Finally, some fungal infections will not respond to antibiotics alone: prosthetic endocarditis, for example, requires combined treatment with reoperation. ${ }^{18}$

1 The British Society for Antimicrobial Chemotherapy and the British Society for Mycopathology. Antifungal therapy. Postgrad Med $\mathcal{F} 1979$; 55:588-700.

${ }^{2}$ Hay RJ. Failure of treatment in chronic dermatophyte infections. Postgrad Med f 1979;55:608-10.

${ }^{3}$ Hurley R, de Louvois J. Candida vaginitis. Postgrad Med $\mathcal{F} 1979$;55:645-7.

4 Symmers W St C. Amphotericin pharmacophobia. Br Med f 1973;iv: 460-3.

${ }^{5}$ Medoff G, Kobayashi GS. Strategies in the treatment of systemic fungal infections. $N$ Engl f Med 1980;302:145-55.

- Atkinson AJ, Bennett JE, Amphotericin B pharmacokinetics in humans. Antimicrob Agents Chemother 1978;13:271-6.

${ }^{7}$ Utz JP, Garriques IL, Sande A, et al. Therapy of cryptococcosis with combination of flucytosine and amphotericin B. F Infect Dis 1975;132 368-73.

8 Bennett JE, Dismukes WE, Duma RJ, et al. A comparison of amphotericin $\mathrm{B}$ alone and combined with flucytosine in the treatment of cryptococcal meningitis. $N$ Engl F Med $1979 ; 301: 126-31$

9 Saunders AM, Goolden AWG, Darrell JH. Cryptococcosis: survival attributed to combination antifungal treatment. $\mathrm{Br} M e d \mathcal{F} 1978$;i: 1930-1.

${ }^{10}$ Hoeprich PD, Ingraham JL, Kleker Elizabeth, Winship MJ. Development of resistance to 5-fluorocytosine in Candida parapsilosis during therapy. 7 Infect Dis 1974;130:112-8.

11 Block ER, Jennings AE, Bennett JE. 5-Fluorocytosine resistance in Cryptococcus neoformans. Antimicrob Agents Chemother 1973;3:649-56.

12 Speller DCE, Fakunle F, Cairns SA, Stephens M. Cryptococcal meningitis complicating systemic lupus erythematosus: two patients treated with flucytosine and amphotericin B. F Clin Path 1977;30:254-61.

13 Vanbreuseghem R, de Vroey C. Systemic opportunistic infections. Postgrad Med F 1979;55:593-4.

14 Speller DCE. Therapy of fungal infections of the central nervous system. Postgrad Med f 1979;55:615-8.

15 Clayton M. Dermatophyte infections. Postgrad Med $\mathcal{F} 1979 ; 55: 605-7$.

${ }_{16}$ Duport B, Drouhet E. In vitro synergy and antagonism of antifungal agents against yeast-like fungi. Postgrad Med $\mathcal{F}$ 1979;55:683-6.

17 Borelli D, Bran JL, Fuentes J, et al. Ketoconazole, an oral antifungal: laboratory and clinical assessment of imidazole drugs. Postgrad Med $\mathcal{F}$ 1979;55:657-61.

18 Wain W, Ahmed M, Thompson R, Yacoub M. The role of chemotherapy in the management of fungal endocarditis following homograft valve replacement. Postgrad Med f 1979;55:629-31.

\section{Investigating constipation}

The Frenchman is obsessed by his liver, the Arab by gases in his stomach, the Englishman by his bowels. From infancy the British are brought up to regard a daily bowel action almost as a religious necessity and to believe in autointoxication from the cesspool of the unemptied colon; so it is little wonder that their doctors see vast numbers of patients obsessed with the frequency, consistency, diameter, and appearance of their stools. Unfortunately, hidden among these hordes, whose lack of bowel actions merely signifies the effects of their modern refined diet or disguises an underlying depression or anxiety state, are occasional exceptions with serious organic disease. How can they be identified?

As so often is the case, a careful history and examination will indicate the answer in most patients with constipation. The commoner organic causes are: obstruction of the lower bowelfor example, by a carcinoma of the colon or by diverticular diseases; painful anal conditions such as fissure in ano; or an adynamic bowel, as seen in Hirschsprung's disease or as the result of taking drugs that decrease the peristaltic activity of the bowel.

The possibility of a carcinomatous stricture of the colon should always be considered when a patient-particularly one over the age of 40 -who has previously had a regular bowel habit develops constipation of increasing severity or, indeed, when a habitually constipated individual finds that his symptoms have become worse without obvious cause. The constipation may alternate with what the patient calls diarrhoea but which is usually the frequent desire to defecate or the passage of mucus in the stool. An obvious warning sign is the presence of fresh or altered blood in the faeces, and the patient may also have abdominal distension, colicky pain, and (usually as late manifestations) loss of appetite, loss of weight, and anaemia.

On abdominal examination a mass may be palpable due to the tumour itself or to faeces impacted above the growth. On rectal examination a tumour in the sigmoid colon may sometimes be felt through the wall of the rectum. An occult blood test on the faeces is often positive. In such cases sigmoido copy is mandatory, and indeed about a third of all growths in the large bowel can be seen with the instrument. Even if the tumour is not seen, suspicion may be aroused if blood or slime is seen in the lumen of the bowel, suggesting a more proximal lesion. The next step is a barium enema examination to clinch or refute the diagnosis. Colonoscopy is being used increasingly as another valuable means of making an exact diagnosis. Diverticular disease of the sigmoid colon can mimic carcinoma exactly in its clinical features. The barium enema examination will usually decide between the two but the radiologist may have difficulty in distinguishing the cause of a sigmoid stricture. Furthermore, these two common conditions may coexist. Less common causes of obstruction include Crohn's disease of the large bowel, a stricture complicating ulcerative colitis, and obstruction due to an extracolonic mass such as a pelvic tumour. Any painful anal condition (such as a fissure in ano or strangulated piles) may inhibit defecation and so precipitate constipation, but a careful local examination should soon establish the diagnosis.

In Hirschsprung's disease the history usually dates to the first month of life. The abdomen is greatly distended and the patient may have eversion of the umbilicus and widening of the subcostal angle. Nevertheless, the diagnosis is occasionally not recognised until adult life. The barium enema examination 